\section{THE SYNTHESIS AND BIOLOGICAL EVALUATION OF SOME NOVEL AMINOHETEROCYCLIC METHOXIME MONOBACTAM DERIVATIVES}

\author{
William J. WheEler*, Don R. Finley \\ and JoHN L. OTT \\ Lilly Research Laboratories, \\ Eli Lilly and Company, \\ Lilly Corporate Center, \\ Indianapolis, Indiana 46285, U.S.A.
}

(Received for publication May 27, 1986)

Two novel monobactams, 3- $\beta$-[2-(3-aminooxazol-4-yl) -2- $Z$-(methoximinoacetamido)]-4- $\alpha$ methyl-2-oxoazetidine-1-sulfonic acid (4) and 3- $\beta$-[2-(5-aminooxadiazol-3-yl)-2- $Z$-(methoximinoacetamido)]-4- $\alpha$-methyl-2-oxoazetidine-1sulfonic acid (5) were synthesized and evaluated microbiologically. Although less active than the corresponding aminothiazole 6 and aztreonam against Gram-negative bacteria 4 was found to be more active than either 6 or aztreonam against Streptococci. The aminooxadiazole 5 was the least active compound tested in this series.

In the past few years, since SQ26445 was isolated from Pseudomonas acidophilia ${ }^{1)}$ and the subsequent synthesis of 3-AMA and the corresponding 4-methyl analogs ${ }^{2)}$, numerous reports concerning the structure-activity relationship of this unique class of $\beta$-lactam antibiotics have appeared $^{3 \sim 5)}$.
Recently several papers concerning the synthesis and microbiological evaluation of novel aminoheterocyclic methoxime cephalosporins have appeared ${ }^{(2)}$. Since the microbiological activity of monobactam antibiotics has been far from optimized, we have undertaken the synthesis of aminooxazolyl- and aminooxadiazolylmethoxime substituted monobactams in hopes of achieving more of a balance between the activities against Gram-positive and Gram-negative bacteria. The results of these investigations are reported herein.

Chemistry

Utilizing HBT-active esters (1 and 2) which have been previously described ${ }^{8, \theta)}$, the 3 -aminomonobactamic acid 3 was acylated under modified Schotten-Baumann conditions (Scheme 1).

\section{Biological Results}

Antimicrobial activities of $\mathbf{4}$ and $\mathbf{5}$ as well as $6^{5)}$ and aztreonam ${ }^{4}$ (included as reference compounds) were determined in an agar dilution assay (Table 1). Activities were determined against a wide variety of Gram-positive and Gram-negative aerobic bacteria (Table 1). All compounds tested were inactive against both penicillin-sensitive and -resistant Staphylococcus aureus as well as Staphylococcus epidermidis. The activities of 4 and 6 were substantially better against Streptococcus pyogenes and Streptococcus pneumoniae than the other compounds tested. While $\mathbf{5}$ was more active than aztreonam, it was 2 to 8 -fold less active than $\mathbf{4}$ and $\mathbf{6}$ against those 2 strains. All of the compounds tested

Scheme 1.

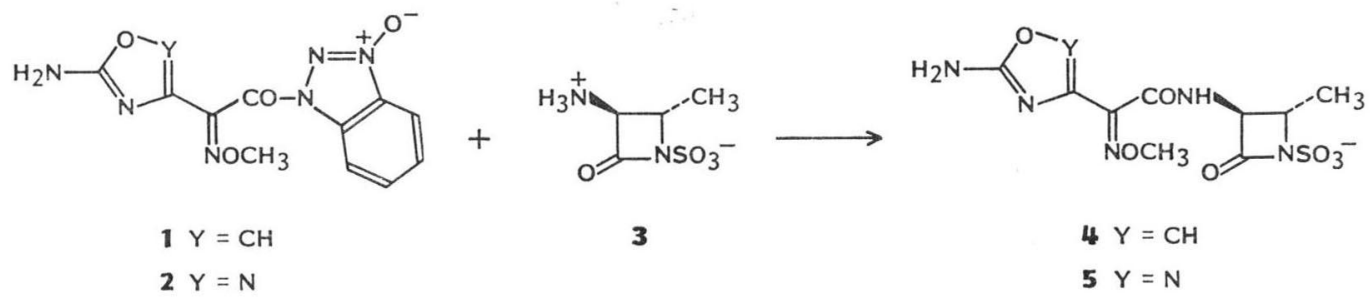<smiles>[R]C(C)(C)C(=O)N[C@H]1C(=O)N([S+](=O)[O-])C(C)[C@@H]1C(=O)O</smiles> 
Table 1. In vitro antibacterial activity.

\begin{tabular}{|c|c|c|c|c|c|}
\hline \multirow{2}{*}{ Species } & \multirow{2}{*}{ Strain } & \multicolumn{4}{|c|}{ Agar dilution MIC $(\mu \mathrm{g} / \mathrm{ml})$} \\
\hline & & 4 & 5 & 6 & Aztreonam \\
\hline Staphylococcus aureus & $\mathrm{X} 1.1$ & $>128$ & $>128$ & $>128$ & $>128$ \\
\hline S. aureus & $\mathrm{V} 41^{\mathrm{a}}$ & $>128$ & $>128$ & $>128$ & $>128$ \\
\hline S. aureus & $\mathrm{X} 400^{\mathrm{a}, \mathrm{b}}$ & $>128$ & $>128$ & $>128$ & $>128$ \\
\hline S. aureus & $\mathrm{S} 13 \mathrm{E}^{\mathrm{a}, \mathrm{b}}$ & $>128$ & $>128$ & $>128$ & $>128$ \\
\hline S. epidermidis & Epi $1^{a, b}$ & $>128$ & $>128$ & $>128$ & $>128$ \\
\hline S. epidermidis & $222^{\mathrm{a}}$ & 128 & $>128$ & 64 & $>128$ \\
\hline Streptococcus pyogenes & $\mathrm{C} 203$ & 2 & 8 & 4 & 16 \\
\hline S. pneumoniae & PARK I & 1 & 8 & 2 & 64 \\
\hline Enterococcus faecalis & X66 & $>128$ & $>128$ & $>128$ & $>128$ \\
\hline E. faecalis & 9960 & $>128$ & $>128$ & $>128$ & $>128$ \\
\hline Haemophilus influenzae & C.L. & 8 & 16 & 0.5 & 0.06 \\
\hline H. influenzae & $76^{\mathrm{c}}$ & 2 & 8 & 0.25 & 0.06 \\
\hline Escherichia coli & N10 & 1 & 32 & 0.06 & 0.125 \\
\hline E. coli & $\mathrm{EC} 14$ & 0.5 & 8 & 0.03 & 0.03 \\
\hline E. coli & TEM $^{\mathrm{c}}$ & 4 & 32 & 0.125 & 0.06 \\
\hline Shigella sonnei & N9 & 1 & 16 & 0.06 & 0.06 \\
\hline Klebsiella pneumoniae & X26 & 1 & 16 & 0.03 & 0.06 \\
\hline K. pneumoniae & $\mathrm{KAE}^{\mathrm{d}}$ & $>128$ & $>128$ & $>128$ & 32 \\
\hline$K$. pneumoniae & X68 & 1 & 16 & 0.06 & 0.03 \\
\hline Enterobacter aerogenes & C32 & 1 & 16 & 0.06 & 0.06 \\
\hline E. aerogenes & EB17 & 1 & 32 & 0.06 & 0.06 \\
\hline E. cloacae & EB5 & 2 & 64 & 0.125 & 0.06 \\
\hline E. cloacae & $265 \mathrm{~A}^{\theta}$ & 64 & 128 & 32 & 32 \\
\hline Salmonella typhi & X514 & 1 & 16 & 0.06 & 0.06 \\
\hline S. typhi & 1335 & 2 & 16 & 0.125 & 0.125 \\
\hline Pseudomonas aeruginosa & $\mathrm{X} 528$ & $>128$ & 128 & 128 & 4 \\
\hline$P$. aeruginosa & $\mathrm{X} 239$ & $>128$ & 128 & 64 & 4 \\
\hline P. aeruginosa & PS18 ${ }^{f}$ & $>128$ & $>128$ & $>128$ & 64 \\
\hline P. aeruginosa & PS72 & $>128$ & $>128$ & 128 & 8 \\
\hline Serratia marcescens & X99 & 4 & 16 & 0.25 & 0.125 \\
\hline S. marcescens & SE3 & 4 & 64 & 0.25 & 0.25 \\
\hline Morganella morganii & PR15 & 8 & 64 & 1 & 0.03 \\
\hline Providencia stuartii & PR33 & 4 & 8 & 0.25 & 0.03 \\
\hline P. rettgeri & $\mathrm{C} 24$ & 0.25 & 8 & 0.03 & 0.015 \\
\hline Citrobacter freundii & CF17 & 1 & 16 & 0.06 & 1 \\
\hline Acinetobacter calcoaceticus & $\mathrm{AC} 12$ & 8 & 32 & 4 & 32 \\
\hline $\begin{array}{ll}\text { a } & \beta \text {-Lactamase producer. } \\
\text { b } & \text { Methicillin-resistant. } \\
\text { c } & \text { TEM (Type } 3 \text { ) } \beta \text {-lactama } \\
\text { d } & \text { Type IVc } \beta \text {-lactamase pr } \\
\text { e } & \text { Constitutive Type } 1 \text { high } \\
\text { \& } & \text { Type Id } \beta \text {-lactamase pro }\end{array}$ & $\begin{array}{l}\text { oducer. } \\
\text { er. } \\
\beta \text {-lactama }\end{array}$ & roducer. & & & \\
\hline
\end{tabular}

were inactive against Enterococci.

Against cephalothin-sensitive Enterobacteriaceae, aztreonam was the most active compound tested. Aminothiazole derivative 6 was usually found to be $1 \sim 2$ dilutions less active against these bacteria, while aminooxazole derivative 4 was $3 \sim 4$ dilutions less active than 6 . The aminooxadiazole derivative $\mathbf{5}$ was significantly less active than any of the compounds tested (MICs ranging from 8 to $64 \mu \mathrm{g} / \mathrm{ml}$ ). This has been the trend observed in the other series examined $\left.^{8}, 0\right) \quad$ (aminothiazole $>$ aminooxazole $>$ aminooxadiazole).

This trend continued when the compounds were tested against the more resistant bacteria. Aztreonam was the only compound which 
showed activity against Pseudomonas aeruginosa, while the activities of aztreonam and $\mathbf{6}$ against resistant Enterobacteriaceae were similar. While 4 possessed useful activity it was generally $3 \sim 4$ dilutions less active than 4 against these bacteria. The activity of $\mathbf{5}$ was significantly less.

\section{Experimental}

NMR spectra were recorded on a Varian Associates EM-390 $(90 \mathrm{MHz})$ spectrometer using tetramethylsilane (TMS) as an internal standard. Chemical shifts $(\delta)$ are reported in parts per million (ppm) relative to TMS. All melting points are uncorrected. Agar dilution MICs were determined by the method described in KIRST et $a l .{ }^{10)}$.

3-[2- $\beta$-(2-Aminooxazol-4-yl)-2- $Z$-methoximinoacetamido]-4- $\alpha$-methyl-2-oxoazetidine-1-sulfonic Acid, Sodium Salt (4)

A $50 \%$ aqueous acetone suspension of $3^{5 \text { ) }}$ $(0.360 \mathrm{~g}, 2 \mathrm{mmol})$ was neutralized to $\mathrm{pH} 6.8$ by the dropwise addition of $1 \mathrm{~N} \mathrm{NaOH}$. The resulting solution was stirred and $\mathbf{1}^{9)}(0.700 \mathrm{~g}$, $2.08 \mathrm{mmol}$ ) was added. Stirring was continued while the $\mathrm{pH}$ of the solution was maintained between 6.8 and 7.0 by the addition of $0.1 \mathrm{~N}$ $\mathrm{NaOH}$. The active ester 1 was slowly consumed and solution was complete after 1 hour, whereupon stirring was continued an additional 2 hours.

The acetone was removed in vacuo and the resulting aqueous solution was washed twice with EtOAc. The aqueous layer was concentrated and crystallization of 1-hydroxybenzotriazole began. After allowing to stand overnight, the solution was filtered and the filtrate was evaporated to dryness. The residue was re-dissolved in warm $\mathrm{EtOH}$ and chilled to effect crystallization. The crystals were filtered to yield 4 as a white solid $(0.1 \mathrm{~g}, 13.6 \%)$, mp 175 $180^{\circ} \mathrm{C}$ (dec). Anal Calcd for $\mathrm{C}_{10} \mathrm{H}_{12} \mathrm{~N}_{5} \mathrm{O}_{7} \mathrm{SNa}$ : C 32.52, H 3.28, N 18.96. Found: C 31.17, H 3.77, N 17.34. NMR (DMSO- $\left.d_{6}\right) \delta 1.46(3 \mathrm{H}$, $\left.\mathrm{d}, J=6 \mathrm{~Hz}, 4-\mathrm{CH}_{3}\right), 3.69(1 \mathrm{H}, \mathrm{dd}, J=3$ and $6 \mathrm{~Hz}, 4-\mathrm{CH}), 3.88\left(3 \mathrm{H}, \mathrm{s}, \mathrm{OCH}_{3}\right), 4.36(1 \mathrm{H}, \mathrm{dd}$, $J=3$ and $7.5 \mathrm{~Hz}, 3-\mathrm{CH}), 4.50\left(2 \mathrm{H}, \mathrm{br} \mathrm{s}, \mathrm{NH}_{2}\right)$, $7.79(1 \mathrm{H}, \mathrm{s}$, oxazole- $\mathrm{H})$ and $9.38(1 \mathrm{H}, \mathrm{d}, J=7.5$ $\mathrm{Hz}, \mathrm{CONH})$.

3-[2- $\beta$-(3-Amino[1,2,4] oxadiazol-4-yl)-2- $Z$ methoximinoacetamido]-4- $\alpha$-methyl-2-oxoazetidine-1-sulfonic Acid, Sodium Salt (5)

A $50 \%$ aqueous suspension of $3^{5)}(0.54 \mathrm{~g}$,
$3 \mathrm{mmol}$ ) was treated as described above with $2^{8)}(1.01 \mathrm{~g}, 3 \mathrm{mmol})$. After work-up, the aqueous layer was evaporated and the residue was dissolved in warm EtOH and filtered. From the filtrate, $\mathbf{5}$ precipitated as a crude amorphous solid $(0.415 \mathrm{~g})$. Crystallization from EtOH yielded 5 as a white solid, $0.153 \mathrm{~g}(13.7 \%), \mathrm{mp}$ $195^{\circ} \mathrm{C}$ (dec). Anal Calcd for $\mathrm{C}_{9} \mathrm{H}_{11} \mathrm{~N}_{6} \mathrm{O}_{7} \mathrm{SNa}$ (EtOH): C 31.71, H 4.08, N 20.18. Found: C $31.47, \mathrm{H}$ 3.76, N. 20.35. NMR (DMSO- $d_{6}$ ) $\delta 1.35\left(3 \mathrm{H}, \mathrm{d}, J=9 \mathrm{~Hz}, 4-\mathrm{CH}_{3}\right), 3.60(1 \mathrm{H}, \mathrm{m}$, 4- $\mathrm{CH}), 3.75\left(3 \mathrm{H}, \mathrm{s}, \mathrm{OCH}_{3}\right), 4.39(1 \mathrm{H}, \mathrm{dd}, J=3$ and $9 \mathrm{~Hz}, 3-\mathrm{CH}), 7.98\left(2 \mathrm{H}, \mathrm{s}, \mathrm{NH}_{2}\right)$ and 9.40 $(1 \mathrm{H}, \mathrm{d}, J=9 \mathrm{~Hz}, \mathrm{CONH})$. In addition the NMR indicated the presence of $1 \mathrm{~mol}$ of $\mathrm{EtOH}$.

\section{References}

1) Sykes, R. B.; C. M. Cimarusti, D. P. Bonner, K. Bush, D. M. Floyd, N. H. GeorgopapadaKou, W. H. Koster, W. C. LiU, W. L. Parker, P. A. Principe, M. L. Rathnum, W. A. SlusarchyK, W. H. Trejo \& J.S. Wells: Monocyclic beta-lactam antibiotics produced by bacteria. Nature 291: 489 491, 1981

2) Floyd, D.M.; A.W. Fritz \& C.M. Cimarusti: Monobactams. Stereospecific synthesis of $(S)$ 3-amino-2-oxoazetidine-1-sulfonic acids. J. Org. Chem. 47: 176 178, 1982

3) Hirose, T.; J. NaKano \& H. Uno: 3-Amino-3methyl-2-azetidinone-1-sulfonic acid deriva ives. J. Pharm. Soc. Jpn. 103: 1210 1214, 1983

4) Breuer, H.; C. M. Cimarusti, T. Denzel, W. H. Koster, W. A. Slusarchyk \& U. D. Treuner: Monobactams. Structure-activity relationships leading to SQ26,776. J. Antimicrob. Chemother. 8 (Suppl. E): $21 \sim 28,1981$

5) Cimarusti, C. M ; D. P. Bonner, H. Breuer, H. W. Chang, A. W. Fritz, D. M. Floyd, T. P. Kissick, W. H. Koster, D. Kronenthal, F. Massa, R. H. Mueller, J. Pluscec, W. A. Slusarchyk, R. B. Sykes, M. TAylor \& E. R. WEAVER: 4-Alkylated monobactams. Chiral synthesis and antibacterial activity. Tetrahedron 39: 2577 2589, 1983

6) Csendes, I.; B. W. Müller \& W. Tosch: Cephalosporin antibiotics. Synthesis and antimicrobial activity of 7 $\beta$-[2-(5-amino-1,2,4-thiadiazol-3-yl)-2-oxyiminoacetamido] cephalosporin derivatives. J. Antibiotics 36: 1020 1033, 1983

7) Teraji, T.: Aminothiazole surrogates. Program and Abstracts of 22nd Intersci. Conf. on Antimicrob. Agents Chemother., Session 35, p. 20, Miami Beach, Oct. 4 6, 1982

8) Wheeler, W. J.; J. B. Deeter, D. R. Finley, M. D. Kinnick, R. Koehler, H. E. Osborne, 
J. T. Ott, J. K. Swartzendruber \& D. G. WISHKA: The synthesis and biological evaluation of $7 \beta$-[2-(5-amino-[1,2,4]oxadiazol-3-yl)-2$Z$-methoximinoacetamido]cephalosporin derivatives. J. Antibiotics 39: 111 120, 1986

9) Wheeler, W.J.; D.R. Finley, R.J. Messenger, R. KoeHler \& J. T. OtT: The synthesis and biological evaluation of 7/-[2-(2-aminooxazol- 4-yl)-2- $Z$-methoximinoacetamido]cephalosporin antibiotics. J. Antibiotics 39: 121 127, 1986

10) Kirst, H. A.; G. M. Wild, R. H. Baltz, R. L. Hamill, J. L. Ott, F. T. Counter \& E. E. OSE: Structure-activity studies among 16membered macrolide antibiotics related to tylosin. J. Antibiotics 35: 1675 1682, 1982 